\title{
Comparative modeling of optical soiling losses for CSP and PV energy systems
}

\author{
Philipp Bellmann \\ Dresden University of Technology, Dresden, Germany \\ Fabian Wolfertstetter \\ German Aerospace Center (DLR), Plataforma Solar de Almeria, Tabernas, Spain \\ Ricardo Conceição \\ Renewable Energies Chair and Institute of Earth Sciences, University of Évora, Portugal \\ Hugo G. Silva \\ Department of Physics and Institute of Earth Sciences, University of Évora, Évora, Portugal
}




\begin{abstract}
Soiling is a challenge for both concentrating solar and photovoltaic technologies. Measurement procedures and efficiency sensitivities to soiling for the same surface particle density differ due to the different optical characteristics of both technologies. For this reason, soiling investigations performed at a site for one technology are not necessarily applicable to the other technology. Soiling measurements have been performed mostly under fixed or rarely occurring angles of incidence. In this study parallel measurements of soiling loss and particle mass density found on the main optical surfaces of concentrating solar power (CSP) and photovoltaic (PV) technologies are presented. The measurements are taken on samples of CSP second surface mirror and PV solar glass with consideration of the main optical characteristics of both technologies. Optical soiling losses are found to be higher by a factor of 8 to 14 in CSP for the same particle surface densities compared to PV. A Mie-based model is presented and validated, that converts the particle mass density and a set of other inputs into the optical soiling loss for either technology for normal angle of incidence and varying angles of incidence. This method facilitates the transfer of soiling loss data from one technology to another. The method can significantly increase the knowledge on soiling for both technologies as more measurement data is made accessible. Additionally, the soiling losses for different angles of incidence can be used to estimate more realistic annual loss parameters for the technologies in question and give recommendations for optimized incidence angles to be used in soiling measurements for both technologies.
\end{abstract}

Keywords: Solar energy, Soiling losses, PV and CSP, Mie scattering, Soiling model, Yield analysis 


\section{Introduction}

The influence of soiling on the production loss of solar devices has been investigated broadly in the past. Concentrating solar thermal power (CSP) and photovoltaic (PV) technologies have in common the preference of sites with high solar resource, where efficiency losses due to low rainfall and accumulation of dust on the optical surfaces tend to be higher. The effect of soiling has been investigated separately for either CSP or PV components in the past. Recent reviews on soiling research [Figgis, 2017; Ghazi, 2014; Kazmerski, 2014; Sarver, 2013; Costa, 2016] provide an overview on these activities. However, there is no study, known to the authors, that compares the effect of soiling losses for both technologies under the same environmental conditions on a measurement or modeling basis. Despite the empirical observation that soiling has more impact on CSP technologies than in PV.

A common experimental approach to investigate soiling is to utilize samples of CSP mirrors or PV cover glass, expose them to artificial indoor or natural outdoor soiling and subsequently measure the broadband specular reflectance for CSP or the transmission for PV [John 2015; Conceição, 2018; Roth, 1980].

The question how soiling influences PV and CSP technology is essentially based on the question how the light interacts with the soiling layer. An important contribution comes from [John, 2015], in which the author investigates the optical losses of artificially soiled glass samples in order to estimate the power loss that would occur in PV systems. The author uses several types of natural dust from different sites in India and applies this dust in a repeatable way to his samples. Relations are given between the particle mass surface density, particle size distribution and the occurring soiling losses. The author concludes that finer particles lead to higher optical losses maintaining the same particle mass surface density.

A significant contribution for CSP technology is given in [Pettit, 1980] through the investigation of wavelength dependent diffuse reflectance and hemispherical reflectance (total reflectance) of soiled mirrors. In that research it is found that most of the light affected by the dust is forward scattered into the mirror, the conclusion is based on the observation that the diffusely reflected light carries the same amount of characteristic wavelength modification as the specularly reflected light, thus must have passed the glass body and the silver layer of the mirror. It is found that only about one fifth of the loss of specular reflectance is due to absorption and almost four 
fifth are lost due to forward scattering. In [John 2015] similar observations are made for moderately soiled PV glass samples and it is found that absorption plays only a minor role. The authors expect that with more intense soiling levels $\left(>2 \mathrm{~g} / \mathrm{m}^{2}\right)$ diffuse reflection of the incoming light by the soiling layer plays an increasing role.

Although Concentrated PV (CPV) systems are out of the scope on this work, some interesting studies on the soiling effect in such technology are mentioned here. In [Stone, 2004] the authors state a $\sim 15 \%$ change in the string current before and after washing, for an exposition period longer than a month. Moreover, interesting studies such as [Montes-Romero, 2019], show an annual mean short-circuit loss around $4.6 \%$, while a loss of $5.7 \%$ was found for the maximum power output. In addition, in [Vivar, 2010], several tests were conducted for different prototypes, such as the Archimedes 2X and Archimedes 10X, which after 5 months of outdoor exposure, presented a loss of around $7 \%$ and $16 \%$, respectively, on the short-circuit current due to soiling. It was inferred by those authors that, soiling has a more severe effect on CPV collectors with higher levels of concentration. This aligns with the observation described before for CSP [Pettit, 1980] that forward scattering is a main mechanism for soiling loss and could be related with an higher soiling impact on CPV than PV.

Returning to CSP and PV comparison, other works investigate the angle of incidence dependency of soiling losses for CSP mirrors [Heimsath, 2016] as well as for PV modules [John, 2015]. For both technologies it is found that the soiling losses increase with higher incidence angles and are minimum at normal incidence. The additional losses due to incidence angle variation for CSP and PV seem to be in a similar range: in [Heimsath, 2016] they are reported to be between 10 to $25 \%$ relative loss of specular reflectance for an incidence angle of $75^{\circ}$ compared to normal incidence, depending on the severity of the soiling for both artificially and naturally soiled mirror samples [Heimsath, 2015]; in [John, 2015] similar values between $10 \%$ to $25 \%$ of relative loss of transmittance are reported for an incidence angle of $75^{\circ}$ compared to normal incidence for different gravimetric densities of artificially soiled glass samples. The intercomparability of those two studies is however not given as the dust particles most probably differ and no values for the gravimetric density are given in [Heimsath, 2015]. The angle of incidence (AOI) dependence of soiling loss influences the effective soiling loss of a power plant, as CSP and PV are both subject to a wide range of incidence angles during the course of a day and year (with the exception of two-axis tracked PV and concentrated Stirling dish systems). 
All of those studies are however only dealing with one of the technologies either CSP or PV individually. It is generally assumed that the losses in CSP are higher than in PV for the same amount of accumulated dust because of the significant differences in the optical pathway as well as optical acceptance and the resulting susceptibility to direct and diffuse light input (specular reflection versus diffuse transmission). Those differences have prior not been investigated in detail and there is no data or models, to the best of the authors knowledge, that provide further insight into this matter. This question is therefore addressed in the present work. Firstly, samples of CSP mirror and PV cover glass are exposed side by side to natural outdoor soiling in southern Portugal for a timespan of several weeks. The samples are collected weekly for measurement of gravimetric particle density and optical losses. The results of this campaign are used to quantify the difference in soiling-induced efficiency losses for CSP and PV technologies. Secondly, the results are used to validate a Mie-based model that derives soiling losses as a function of particle size distribution, particle mass and incidence angle. The model is based on the assumption that all intercepted light is either reflected, absorbed or scattered and that this has a unique effect on each CSP and PV due to their significant differences in optical behavior. Finally, recommendations on the incidence angles that best represent the angles occurring during operation of commercial plants are given.

\section{Experimental Methods}

\subsection{Soiling exposure}

Twenty-five solar mirror and glass samples each were exposed outdoors on a structure shown in figure 1 under various tilt angles $\left(0^{\circ}, 15^{\circ}, 30^{\circ}, 45^{\circ}, 60^{\circ}, 75^{\circ}\right.$ and $\left.90^{\circ}\right)$ oriented to the cardinal directions - the structures were provided by authors, Conceição and Silva, and described in [Conceição, 2019]. The exposure lasted approximately 9 weeks and took place in an agricultural setting in rural Portugal on an experimental site (Valverde) of the University of Évora, approximately $10 \mathrm{~km}$ outside of the city during a dry and hot summer with temperatures regularly exceeding $40{ }^{\circ} \mathrm{C}$ and relative humidity dropping below $10 \%$ during daytime. The glass samples were left uncleaned during the whole period. The mirror samples were cleaned once 
after approximately 4 weeks. This is consistent with the common practice of rarely cleaning PV and frequently cleaning CSP devices. For this work only, data from the south-facing samples is used. The devices used to measure the optical soiling loss of glass and mirror samples introduced in Section 2.2 - both have a default sample installed which is as well exposed to the same outdoor conditions, with the main difference being that both are installed on a Sun Tracker device.
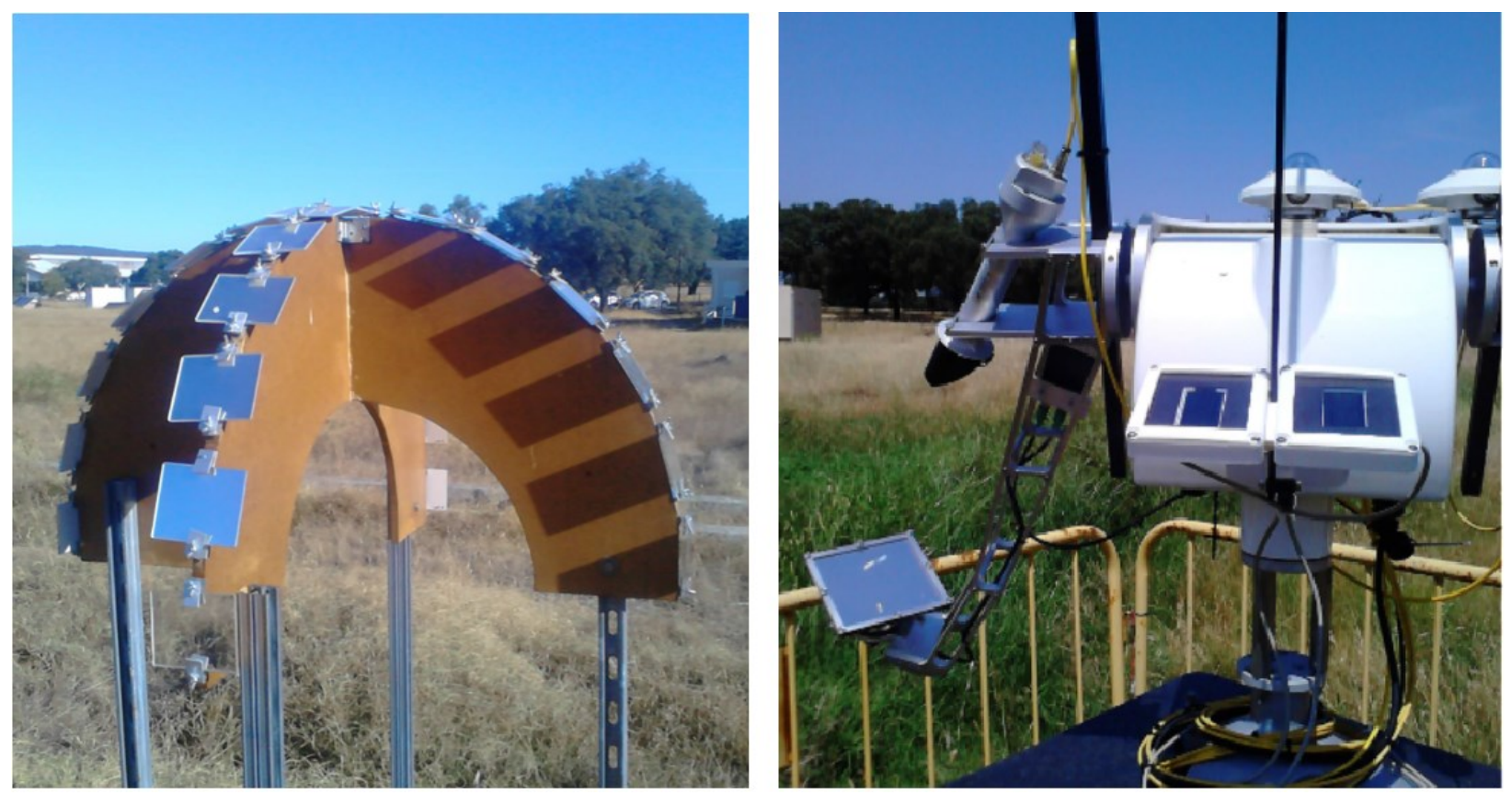

Figure 1: Outdoor soiling exposition for (fixed) CSP mirror and PV glass samples (left). Measuring devices for CSP and PV soiling loss and (tracked) exposure of their default samples on a Sun Tracker (right).

\subsection{Measurement of optical loss}

The loss of efficiency in energy conversion specific to the technology, CSP or PV, caused by soiling on the main optical surfaces can be expressed in good approximation through the efficiency factor "cleanliness", $\xi$, which is defined as one for a perfectly clean mirror or glass and zero for absolutely soiled ones. Cleanliness is measured using two devices for the two types 
of samples (mirrors and glasses) and, as mentioned before, both devices are mounted on a Sun Tracker and use the Sun as natural light source (see also Figure 1)

Cleanliness for PV is determined as the ratio of short-circuit current of a soiled and a clean solar cell. The short-circuit current is assumed to be directly proportional to the loss of energy produced by a PV solar field. There is a small degree of inaccuracy involved in this assumption due to temperature differences between clean and dirty panels, inhomogeneity of soiling patterns on modules, etc. For CSP, the optical efficiency loss is assumed to be directly proportional to energy yield, which is only completely true if increased heat losses due to higher heat transfer fluid temperatures or differences in turbine ramp-up and -down are neglected. As these inaccuracies are lower than the effect of soiling by some percentage points, these assumptions are considered valid for this study.

\subsubsection{TraCS - Tracking Cleanliness Sensor}

Tracking Cleanliness Sensor (TraCS) uses two pyrheliometers mounted on a Sun Tracker. The first one measures a reference direct-normal irradiance (DNI, $I_{0}$ ) coming directly from the sun. The second one measures the specularly reflected DNI $\left(I_{R}\right)$ from a soiled solar mirror sample which is as well attached to the sun tracker (on a rotating mount to extend the measured area on the sample). The ratio of $I_{R}$ and $I_{0}$ results in the reflectance, $\mathrm{R}$, of the mirror. Cleanliness is calculated, for time $t$, by $\xi(t)=R(t) / R_{0}$, in which $R_{0}$ is a constant used to guarantee that $\xi$ is one in the beginning of the experiment (both mirrors clean). See [Wolfertstetter, 2012] for more information on the device and methodology. A continuous data set with 1-min time steps is recorded using TraCS and its factory made mirror sample, over the period of the experiment. This data set is processed using the MatLab robust LOWESS algorithm for excluding outliers and smoothing. The processed data is subsequently averaged over solar noon $\pm 2 \mathrm{~h}(\approx 10 \mathrm{am}$ to $2 \mathrm{pm}$ ) to obtain a daily mean value. Cleanliness of the mirror samples exposed on the device shown in figure 1 is measured on a weekly basis using TraCS as reflectometer. This is achieved by swapping the factory sample with the ones to be measured. For each of those samples a 2-min data set with $10 \mathrm{~s}$ time step is recorded and averaged. These measurements were taken under conditions of clear sky $\left(\mathrm{DHI}^{1} / \mathrm{GHI}^{2} \leq 0.1\right.$ and $\left.\mathrm{DNI} \geq 500 \mathrm{~W} / \mathrm{m}^{2}\right)$.

\footnotetext{
${ }^{1}$ DHI stands for diffuse horizontal irradiance.

${ }^{2} \mathrm{GHI}$ means global horizontal irradiance. 


\subsubsection{PvSM - Photovoltaic Soiling Monitor}

The PvSM makes use of two polycrystalline (c-Si) solar cells underneath two PV cover glass samples, all mounted on the same Sun Tracker. One glass sample is cleaned on a daily basis whereas the other is left to natural soiling. The short-circuit current $I_{S C}$ and the temperature, $T_{c}$, of both cells is measured continuously with 1-min time steps. The short-circuit currents of the cells are then temperature corrected according to [Knisely, 2013]. The ratio of the temperature corrected short-circuit currents $I_{\mathrm{SC} 2}$ and $I_{\mathrm{SC} 1}$ results in the transmittance, $T$, of the glass. Cleanliness is calculated, for time $t$, by $\xi(t)=T(t) / T_{0}$, in which $T_{0}$ is a calibration factor found at the beginning of the experimental campaign to make up for slight deviations in the used shunt resistors and PV cells, thus to calibrate the Cleanliness value to one for clean glasses. This is the common principle of measuring PV soiling losses, and has been validated in the literature [Casanova, 2012; Ryan, 1989; Caron, 2013]. Similar to the mirror samples the Cleanliness of the glass samples is measured weekly by swapping the default sample of the PvSM with the sample from the depositor to be measured. Also, here a 2 min data set with $10 \mathrm{~s}$ time step is recorded and averaged. The measurements are taken at the same time of the weekly measurements in section 2.2.1 and thus under the same conditions. An uncertainty analysis of the measurements using the PvSM considering the uncertainty of the dataloggers input stage, the variations of the components (PV cells, shunt resistors, temperature sensors) and the change in solar spectrum results in an overall relative uncertainty of $\pm 1 \%$ of the measured Cleanliness.

\subsubsection{Measurement of gravimetric density}

Gravimetric density of soil on the mirror and glass samples is calculated from weight measurements and the surface area of the samples. The weight measurements are done on a weekly basis preferably on the same day on which the optical measurements are carried out. The samples are kept in a room with controlled temperature and a dehumidifier, over night before the measurement to ensure minimal influence of humidity on the soil weight. The back of the samples is cleaned before drying to ensure only dust on the front side is contributing to the measurement. The reference weight of each (clean) sample is measured before the experiment after cleaning the samples with deionized water and a lint-free cloth. 


\section{Experimental Results}

\subsection{Soiling rate}

The continuously recorded data of Cleanliness $\xi$ for both TraCS and PVSM shows that the soiling rate is 8 to 9 times higher for the TraCS mirror sample $(\approx 0.35 \% /$ day $)$ compared to the PvSM cover glass sample $(\approx 0.04 \%$ /day) as shown in figure 2 . The samples of both devices are exposed to outdoor soiling in the same way in respect to position, orientation, movement on the sun tracker and duration of the exposure. The figure indicates the manual cleaning of the mirror sample after approximately 4 weeks of exposure to ensure that the reflectance is kept above approximately $90 \%$ according to [Cohen, 1999]. Data for the location under study, shows that the soiling effect on mirrors reflectance loss is about 4 times lower than, for instance, in Benguerir Morocco [Conceicão, 2019], and thus a lower cleaning frequency, which is about every two weeks for such dusty locations, is expected. The glass sample remains uncleaned during the whole period.

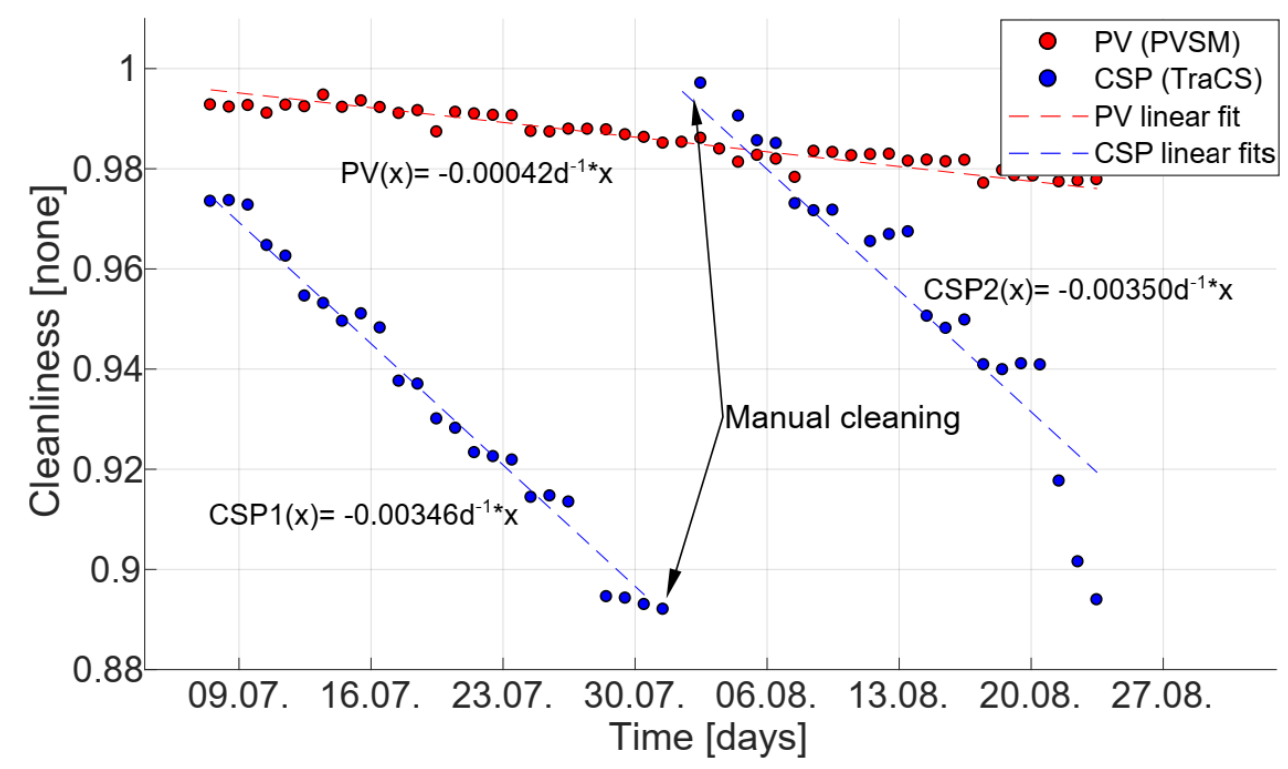

Figure 2: Comparison of the daily mean values of Cleanliness continuously measured with the default samples of TraCS and PvSM - and linear fits whose slopes are interpreted as mean soiling rate per day. The samples of both devices are exposed to outdoor soiling in the same way in respect to position, orientation, movement on the sun tracker and duration of the exposure thus are expected to have nearly the same gravimetric density. 


\subsection{Soiling loss versus gravimetric density}

The data set of weekly measurements of relative optical loss versus gravimetric density suggests that the optical soiling loss of the mirror samples is approximately 8 to 14 times higher than the loss of the corresponding glass samples with the same gravimetric density as shown in figure 3 . Each week data points are marked with a specific color and linear functions have been fitted to the data of each week. The mentioned factors 8 and 14 result from comparing the maximum and minimum steepness of the linear fits for both graphs. For these low gravimetric density levels $\left(\rho_{\mathrm{g}}\right.$

$\leq 1 \mathrm{~g} / \mathrm{m}^{2}$ ) that were reached during the experiment the correlation between optical loss and gravimetric density has a linear tendency when considering the data of each week separately. This linear behavior has been reported before [John, 2015; Sarver, 2013; Sayyah, 2014]. The soiling losses for the same gravimetric density tend to rise over the duration of exposure for the mirrors and to fall over time for the glasses. This is interpreted to be an effect of a change in size distribution of the soiling layer over time as is reported in [Roth, 1980]: The relative number of smaller particles deposited on an uncleaned surface increases over time in a natural outdoor soiling setting, thus possibly leading to higher scattering and lower shading/absorption effects. This is because scattering is the dominant mechanism for light loss at particle layers as compared to absorption. The results of the experiments lead to the hypothesis that forward scattering is the main interaction of the incoming light with the soiling layer for low soiling levels. CSP devices would suffer most soiling losses from forward scattering outside of their acceptance angle, where PV devices can still utilize most of the forward scattered light as it is scattered within their (much wider) acceptance angle. Figure 3 shows the proposed behavior in a simplified way: the incoming irradiance $I_{0}$ is partly absorbed $\left(I_{A}\right)$, backwards scattered or reflected diffusely $\left(I_{R}\right.$ diff $)$. The useful portion for CSP (left) is the specularly reflected light $\left(I_{R}\right)$ only, whereas PV (right) converts direct and diffuse transmitted light $\left(I_{T \text { direct }}\right.$ and $\left.I_{T \text { diffuse }}\right)$. 
a) Mirrors

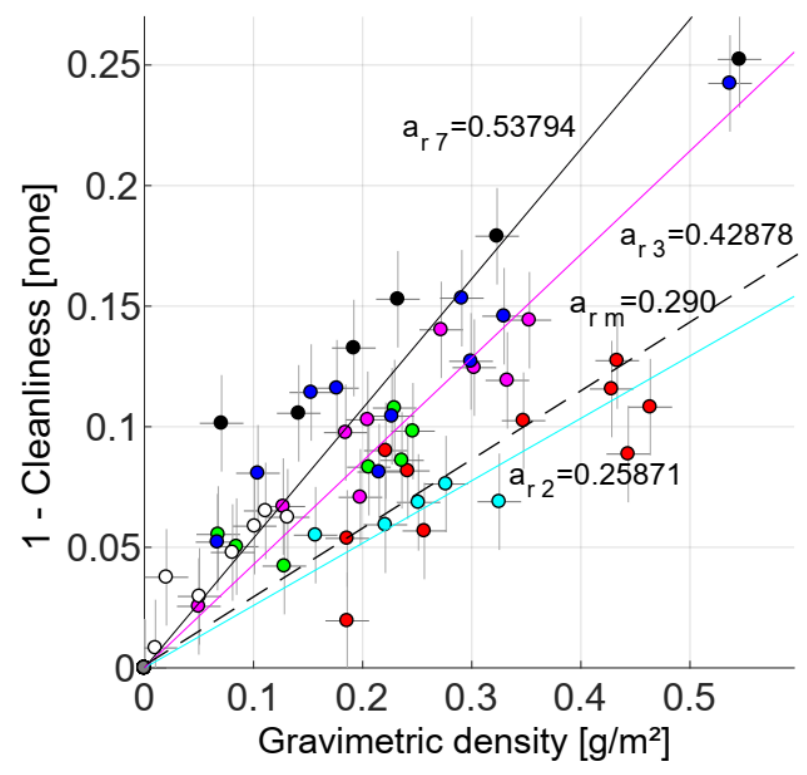

b) Glasses

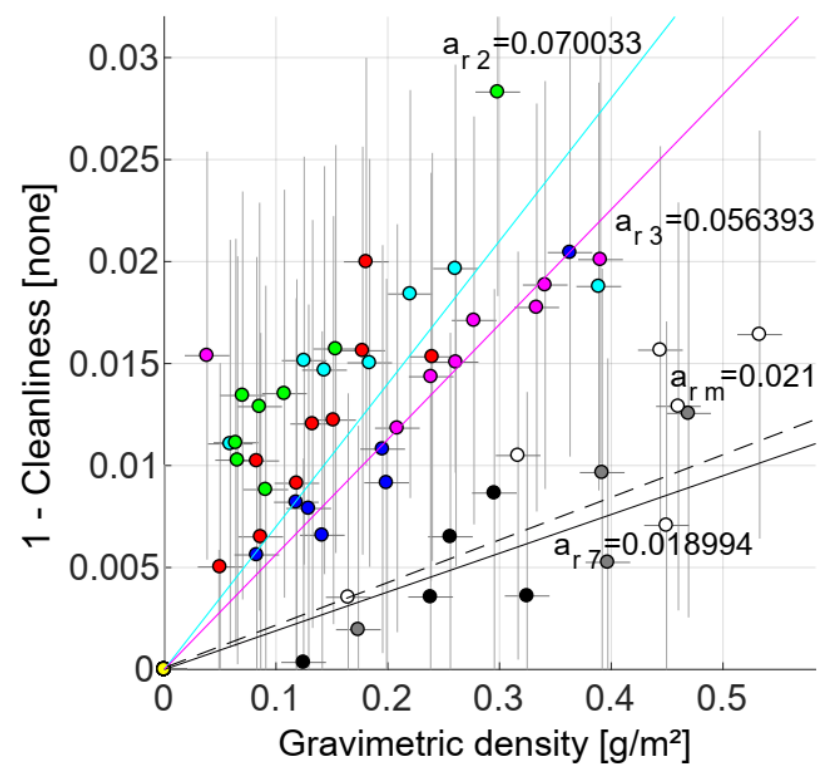

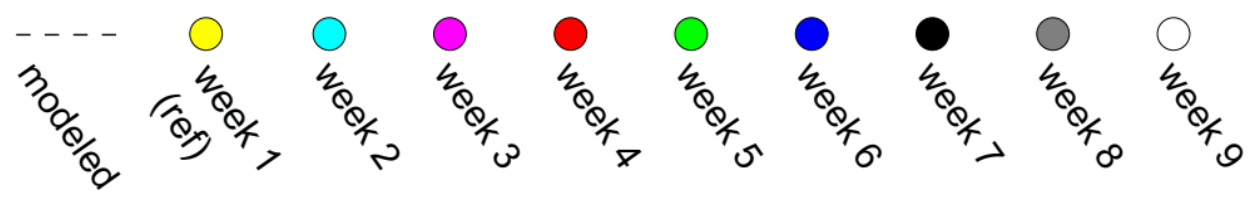

Figure 3: Plots of gravimetric density versus Cleanliness (=efficiency loss) from the southfacing outdoor soiled mirror and glass samples (depositor) across the weeks of the experiment, and linear fits for the data of the $2^{\text {nd }}, 3^{\text {rd }}$ and $7^{\text {th }}$ week. The black dashed line shows the modeled data introduced later in section 4.4. Note the difference of y-axis scaling.

\section{Model}

\subsection{Assumptions}

Based on the experimental results and their interpretation, a physical model is compiled based on Mie-scattering theory. The model essentially assumes a partial scattering and absorption of the incoming light that interacts with the soiling particles. The absorbed light is fully lost for both technologies CSP and PV. The scattered light is either scattered within or outside the optical acceptance of the corresponding technology and thus either usable or lost for energy conversion. The model is based on the following assumptions:

1. Particles are considered as spherical; 
2. The size distribution is taken from [Figgis, 2016] for a PV dust sample from Qatar;

3. The complex refractive index for Saharan dust is taken from [Wagner, 2012];

4. Only single interactions of particles with light are assumed;

5. Low soiling levels $\left(<2 \mathrm{~g} / \mathrm{m}^{2}\right)$;

6. One interaction with soiling layer for glasses, two full interactions for mirrors (no double shading);

7. Normal incidence angle $\theta=0^{\circ}$.
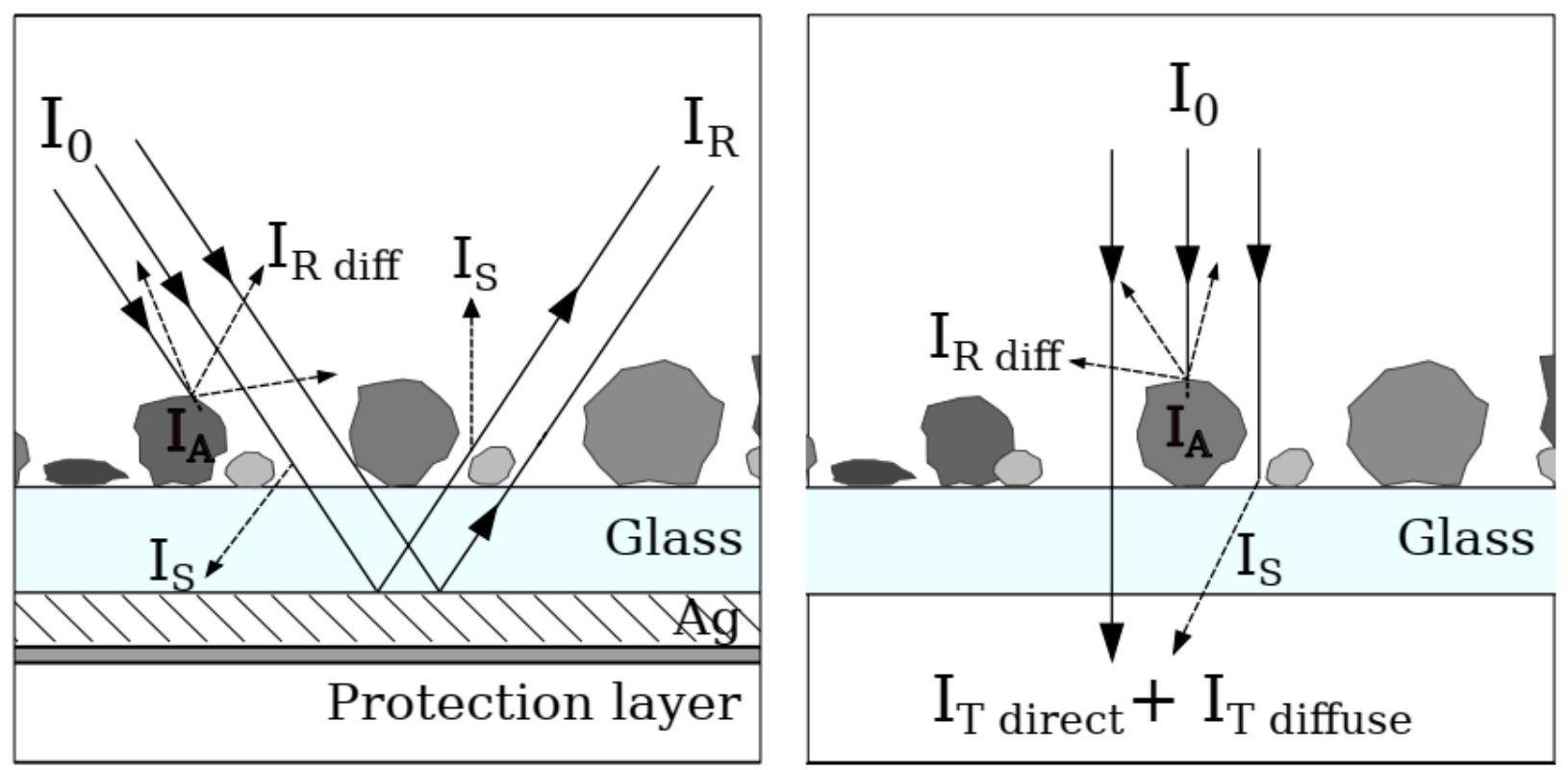

Figure 3: Interaction of irradiance with soiling particles on the surfaces of CSP mirrors (left) and PV glasses (right): The incoming irradiance I0 is partly absorbed (IA), backwards scattered or reflected diffusely (IR diff). The useful portion for CSP (left) is the specularly reflected light (IR) only, whereas PV (right) converts direct and diffusely transmitted light (IT direct and IT diffuse).

It should be noted that during Spring and beginning of Summer, there is an increase organic soiling, namely pollen, such as Arecaceae; Pinaceae; Quercus sp and Poaceae. The rest of the year, the deposited matter is mainly mineral, such as iron oxides, aluminosilicates and halite. The latter can arrive at this location due to evaporation of sea spray when wind speed and direction are favorable [Conceição et al., 2018; Conceicão_Thesis, 2019]. The pollen found for this region had 20 to $30 \mu \mathrm{m}$ in diameter, while mineral matter aggregates had between 14 to $15 \mu \mathrm{m}$ 
diameters. Smaller mineral matter, including submicron scale, was also found in SEM imagery [7]. For this model, particles are considered to be homogeneously distributed on the surfaces.

Due to different types of deposited soiling (optical properties of the particles change overtime due to the different sources of them), dew occurrence, as well as, measurements deviations, some scattering can be seen in Fig. 4.

\subsection{Description}

The model calculates a value of Cleanliness $\xi$ based on the following inputs: gravimetric density, size distribution and complex index of refraction of the soiling particles as well as spectral response and acceptance angle response of the respective technologies. The light-red highlighted area in the flowchart, shown in Figure 5, that shows the basic principle of the model: the optical losses are subtracted from the incoming irradiance. Absorption losses are subtracted fully, scattering losses only partially according to the optical acceptance characteristics of either technology. For mirrors the interaction with the soiling layer occurs twice, thus a square operation is carried out in the CSP case. Equations 1 and 2 describe the operations underlying this basic concept mathematically. The operations and inputs around these equations are further explained: the conversion of gravimetric density, $\rho_{\mathrm{g}}$, to percentage of area covered by the particles, $\mathrm{k}_{\mathrm{a} \text { cov }}$, assumes spherical particles, the mentioned size distribution and no conglomeration of particles. It is the ratio of the summed up square sections of the soiling particles, "shadow", to the surface area of the sample. The percentage of incoming light that is scattered and absorbed results from multiplying $k_{a}$ cov with the efficiencies for scattering and absorption, $Q_{\text {scat }}$ and $Q_{a b s}$, respectively. These efficiencies are derived using MiePlot [Laven, 2017] which outputs them as a function of the wavelength $\lambda$. Those functions $Q_{i}(\lambda)$ are weighted with the effective spectrum of the respective technology. The effective spectrum meaning here: the portion of the solar spectrum which is actually converted into usable energy - after passing through all optical components - in a CSP device (parabolic trough [Wolfertstetter, 2016]) or a PV device (generic polycrystalline silicon module [Knisely, 2013], including Si spectral response). The information about which percentage of the scattered light (scattering angle $\beta$ ) is scattered within or outside of the optical acceptance angle of the technology is derived by using 
the cumulated probability density functions $\operatorname{CPDF}(\beta)$ output from MiePlot and weighting them with the acceptance angle response function $W(\beta)$ of either technology. The $\operatorname{CPDF}(\beta)$ are integrated and normalized forms of the scattering intensity versus scattering angle functions $\left|S_{j}(\beta)\right|^{2}$. In the case of CSP the acceptance angle response of the EuroTrough [Wolfertstetter, 2016] is used for $W_{C S P}(\beta)$, in case of PV the AOI response of a generic polycrystalline silicon module [Knisely, 2013] is used for $W_{P r}(\beta)$.

$$
\begin{gathered}
C_{C S P}=\left\{1-k_{a c o v} \cdot\left[Q_{a b s}^{C S P}+Q_{\text {scat }}^{C S P} \cdot\left(1-\int_{0^{\circ}}^{180^{\circ}} \frac{d C P D F(\beta)}{d \beta} \cdot W_{C S P}(\beta) d \beta\right)\right]\right\}^{2} \\
C_{P V}=1-k_{a c o v} \cdot\left[Q_{a b s}^{P V}+Q_{s c a t}^{P V} \cdot\left(1-\int_{0^{\circ}}^{180^{\circ}} \frac{d C P D F(\beta)}{d \beta} \cdot W_{P V}(\beta) d \beta\right)\right]
\end{gathered}
$$

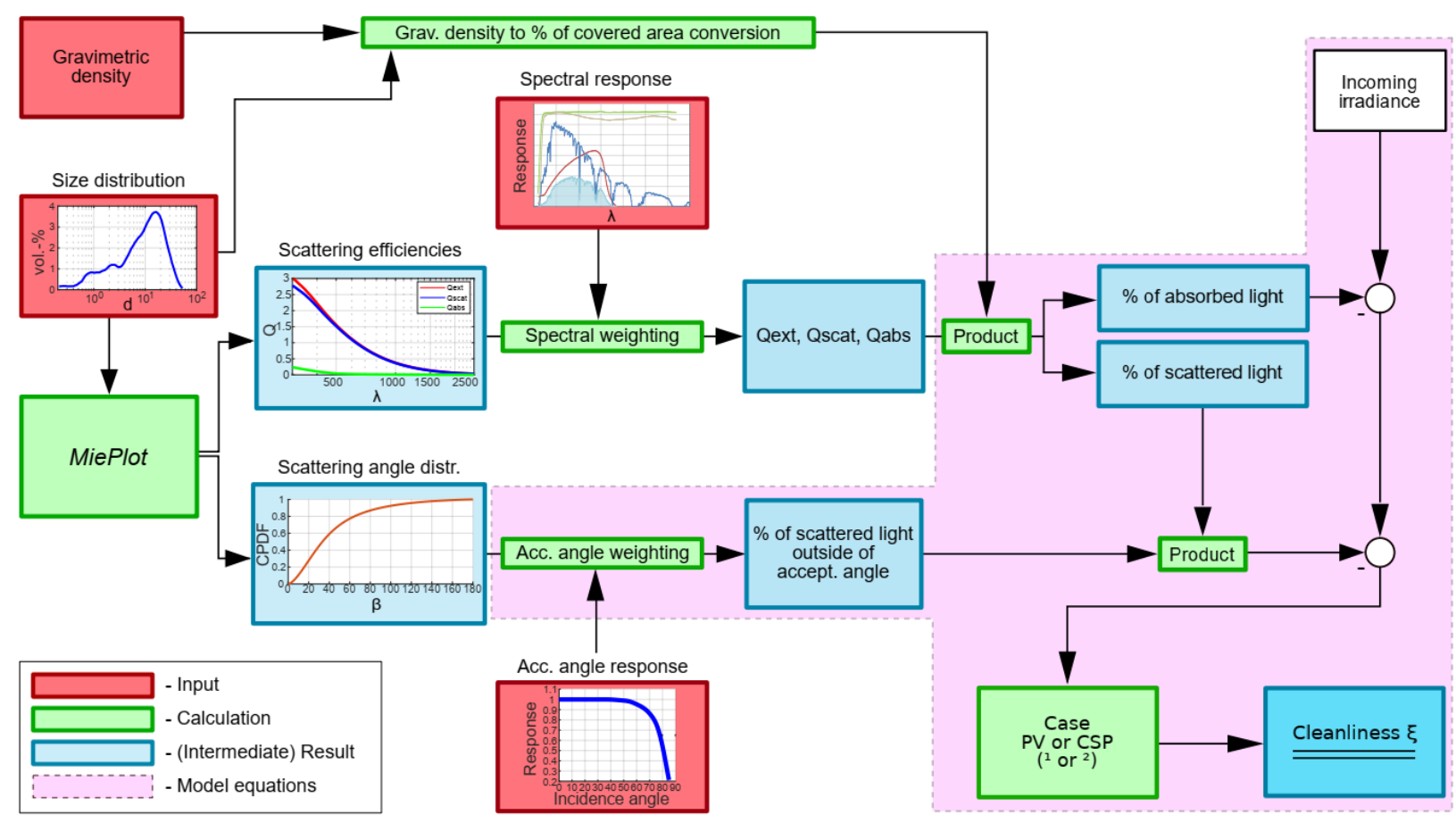

Figure 5: Flowchart of the structure of the model that predicts Cleanliness values on the basis of the gravimetric density and a set of specific input parameters shown in red. The light-red highlighted area represents the operations carried out by the model equations 1 and 2 . 


\subsection{Extension for non-normal incidence}

The model is extended experimentally for non-normal angles of incidence for PV devices. This extension assumes that the scattering happens with rotational symmetry and therefore cones of scattered light rays of equal light intensity intersect the module surface as depicted in figure 6 . Each ray reaches the surface with a specific angle $\theta_{\text {ray }}$ which is a function of the angle of incidence $\theta$ (cone axis), the scattering angle $\beta$ (between cone axis and cone surface) and the position of the ray on the cone surface (angle of rotation $\gamma$ ). This ray-specific angle is then used to weigh the intensity of the ray with the acceptance angle response of a PV module.

Although the resulting graphs show artifacts for incidence angles $>80^{\circ}$ and are not fully consistent with the observations made in e.g. [Martín, 2002; Martan, 2012] they are used for further calculations. The error made by this assumption is acceptable, because the contribution to annual PV production is small for large incidence angles, compare figure 8 below.

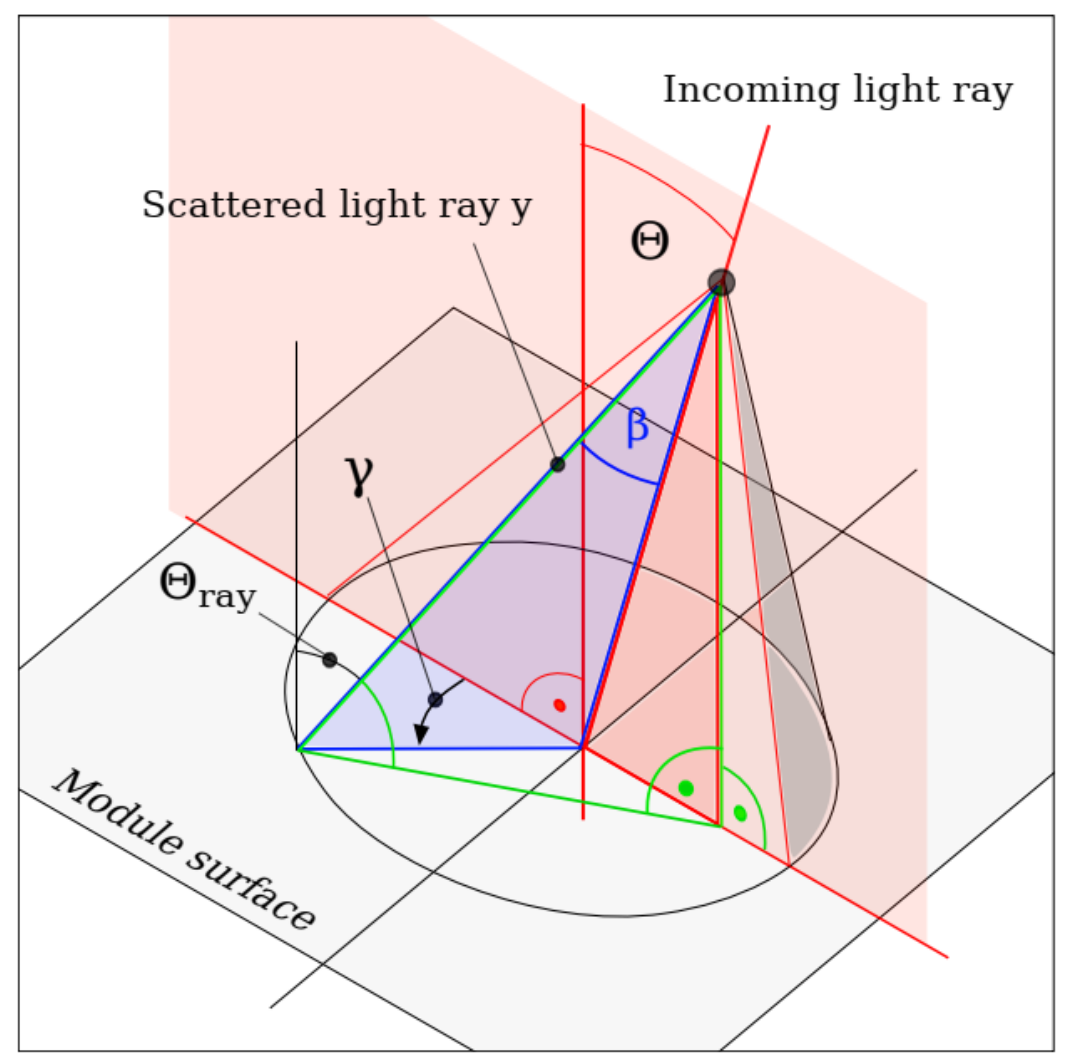

Figure 6: Each scattered light ray reaches the module surface with the angle $\theta_{\text {ray }}(\theta, \beta, \gamma)$ and is weighted with the optical acceptance characteristics of a PV module. 


\subsection{Data modelled versus measured}

The model output is compared with the results of the experiments. The experiments showed a range of slopes for linear fits for relative loss versus gravimetric density for both technologies $\mathrm{CSP}$ and PV. This range can be approximated through the minimum and maximum slopes of the linear fits shown in figure 4 . The model output can be similarly expressed as a linear function of relative loss versus gravimetric density. For the assumptions and input parameters stated in Section 4.1 the output of the model is compared to the linear fits from figure 4 . The output lies within the range of the measured data points for both technologies, as is shown in figure 4 . The trend of the model for rather underestimating soiling losses are thought of to be a result of the various assumptions that were made; specifically, the assumed constant size distribution, the considered optical properties of the dust, the approximation of spherical particles, the no occurrence of agglomeration of dust particles.

\section{Influence of AOI on Annual Cleanliness}

It has been shown that non-normal AOIs lead to increased soiling losses [Martí, 2002; Heimsath, 2016] compared to normal AOI. Therefore, it is an interesting topic to bring Cleanliness and soiling loss measurements into the context of non-normal AOIs. The AOIs that occur during the operation of both CSP and PV devices throughout the year offer the possibility to calculate an overall annual Cleanliness in dependence of the AOI behavior of the devices.

\subsection{Annual energy weighted angles of incidence}

To investigate how severe the varying AOIs in a power plant can influence the overall Cleanliness during a whole year, the first step is to calculate the occurring AOIs with their corresponding occurrence frequency and weights based on the energy input at this angle.

A calculation is done that estimates annual energy weights for all incidence angles for both a north-south oriented CSP parabolic trough and a south-facing stationary PV module with $30^{\circ}$ tilt angle. The trough calculations considered that the bent surface of the trough naturally leads to a variety of AOIs in each given situation, based on EuroTrough geometry. For the PV calculations a separation between direct and diffuse irradiance is made that is based on an even distribution of 
diffuse light across the sky, while neglecting reflections from the ground. The results are shown in figure 7.

Both CSP and PV relative contributions are calculated for the location of Évora, Portugal (Southern Europe), using weather data from 2016-8-17 to 2017-8-16 measured on site. Only irradiance values of DNI $>200 \mathrm{~W} / \mathrm{m}^{2}$ and $\mathrm{GTI}^{3}>50 \mathrm{~W} / \mathrm{m}^{2}$, were considered for CSP and PV, respectively, to account for low system efficiencies at low irradiance levels [Janotte, 2012; Baltus, 1997]. CSP angles consider the manifold of occurring incidence angles in a parabolic trough at each given moment as a result of the bent surface of the reflector. PV angles consider a separate weighting for direct and diffuse irradiance contribution. Reflections from the ground are neglected. The $\mathrm{x}$-axis specifies upper bin limits, i.e., $0^{\circ}$ to $5^{\circ}$ for the left-most bar.
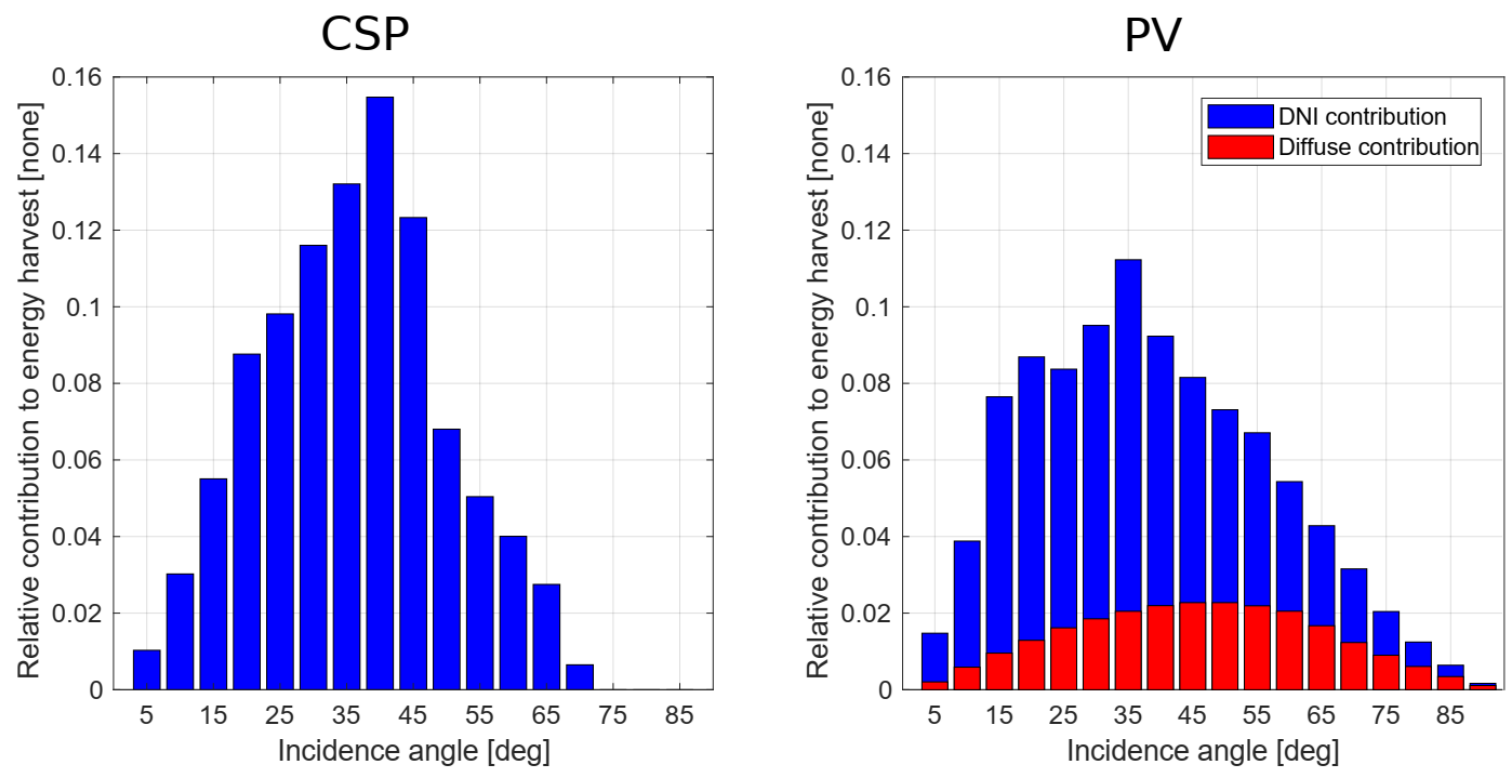

Figure 7: Annual energy weighted incidence angles for: (left plot) an EuroTrough parabolic trough in north-south orientation (left); (right plot) a PV module with $30^{\circ}$ tilt, south orientation (right).

\subsection{Soiled AOI responses}

For the comparison of AOIs influence on Cleanliness values in an annual context two cases are investigated: case 1- assumes the same normal AOI Cleanliness value $(\xi=0.95)$ for both CSP and PV; case 2 - assumes the same gravimetric density $\left(\rho_{g}=0.3 \mathrm{~g} / \mathrm{m}^{2}\right)$ for both CSP and PV.

${ }^{3}$ GTI is an abbreviation for Global Tilted Irradiance. 
The corresponding soiled PV AOI responses are extracted from the extension of the model described in Section 4.3. The soiled CSP AOI responses are calculated using the model proposed in [Heimsath, 2016]. The most obvious difference in AOI behavior of both technologies is that the CSP curves show a much shallower roll-off for higher AOIs compared to the PV curves. According to the investigation done in [Heimsath, 2016] CSP mirrors do not show any or very little loss for high AOIs in their clean state. PV devices on contrary do show significant losses for higher AOIs starting from around $60^{\circ}$ even in clean state [Knisely, 2013].
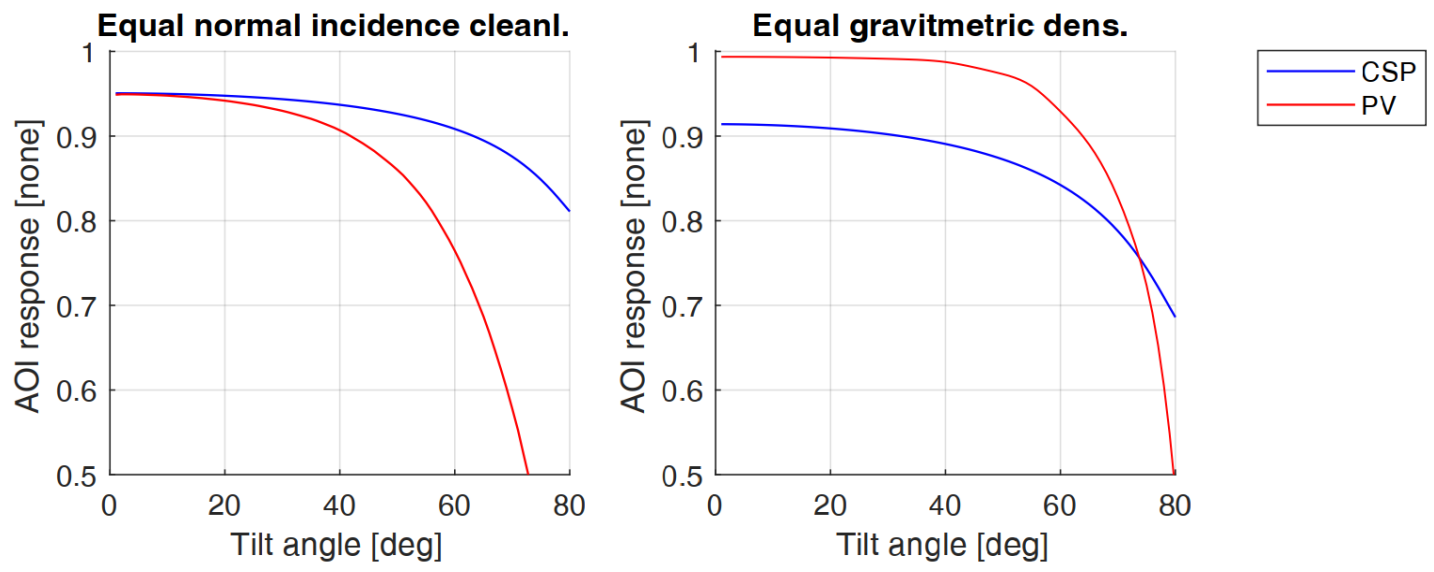

Figure 8: AOI responses used for annual cleanliness calculations: (left plot) case 1 - equal normal incidence Cleanliness $C=0.95$; (right plot) case 2 - equal gravimetric density $\rho_{g}=0.3$ $\mathrm{g} / \mathrm{m}^{2}$.

\subsection{Influence on annual Cleanliness}

Using the AOI responses of soiled PV modules and soiled CSP mirrors as well as the energy weighted annual AOIs from Section 5.1 an annual Cleanliness can be estimated. This is then compared with the Cleanliness measured at (near) normal incidence. Each energy weighted annual AOI is multiplied with the corresponding soiled AOI response, summed up and referenced to the annual clean state, considered as reference yield. The annual clean state reference for CSP is one whereas it is 0.968 for PV as shown in the leftmost plot in figure 9, which is due to the fact that clean mirrors have virtually no AOI losses [Heimsath, 2016]. Significant differences between the Cleanliness measured under (near) normal incidence and the corresponding annual values are shown. The tendency for higher annual losses compared to the normal incidence case is stronger for PV than for CSP devices. CSP shows higher overall losses for the same level of soiling. It is shown that Cleanliness measurements taken under normal AOI 
do not necessarily reflect the optical losses well that are induced on the plant by the corresponding level of soiling, considering the range of occurring AOIs during the whole year.

\subsection{Recommendations for measuring Cleanliness under various tilt angles}

The energy weighted incidence angles introduced in 5.1 are binned into coarser bins. The result is a set of angles and corresponding weights shown in table 1 that can be used to measure the Cleanliness under various AOIs to reflect the actual plant losses due to soiling more accurately than sole (near) normal incidence Cleanliness measurements. The differences between using the proposed method to a sole normal incidence measurement has been explained in Section 5.3 and shown in figure 9.

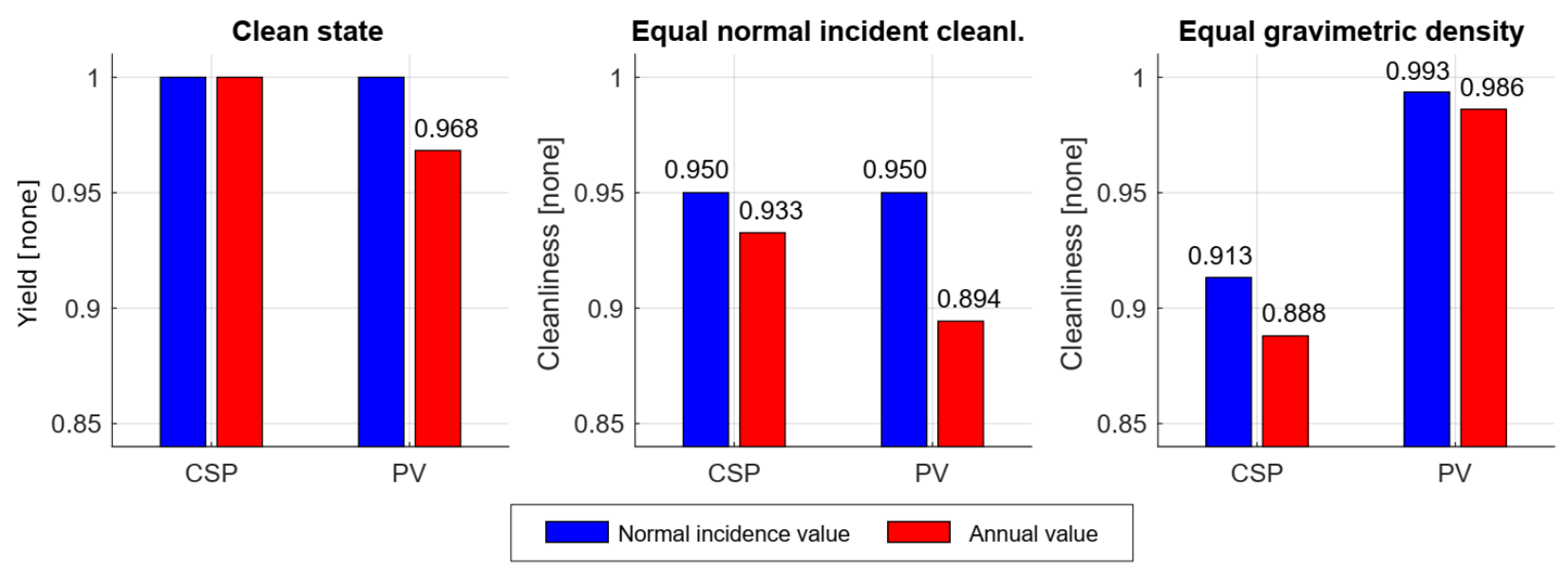

Figure 9: Annual cleanliness values compared to values measured for normal incidence: (left plot) shows the assumption made that a clean mirror shows no AOI losses whereas even a clean PV panel shows considerable AOI losses - the yield factors of the clean state are used as reference to calculate the annual Cleanliness; (middle plot) case 1 - shows the difference of AOI influences between both technologies - PV suffers higher soiling losses throughout the year than CSP due to a stronger attenuated AOI response for high incidence angles; (right plot) case 2 shows the difference in influence of the same gravimetric density on both technologies.

Table 1 Suggestions for measuring cleanliness under various incident angles with corresponding weights to account for the incidence angle distribution during a year of plant operation and derive a more representative Cleanliness value than the usual normal or near-normal incidence. Values rounded within $\pm 3^{\circ}$ respectively $\pm 2 \%$. 


\begin{tabular}{|l|l|l|l|}
\hline \multicolumn{2}{|l|}{ CSP } & PV \\
\hline AOI $\left[^{\circ}\right]$ & Weight $[\%]$ & AOI $\left[^{\circ}\right]$ & Weight $[\%]$ \\
\hline 15 & 20 & 15 & 30 \\
\hline 30 & 50 & 35 & 40 \\
\hline 50 & 25 & 55 & 25 \\
\hline 60 & 5 & 75 & 5 \\
\hline
\end{tabular}

\section{Conclusions}

The optical soiling losses in the main optics for both technologies CSP and PV have been investigated in detail in direct comparison. It has been shown that the same amount of dust (soiling gravimetric density) results in an approximately 8 to 14 times higher soiling loss for CSP technologies compared to PV. It has been shown that these soiling losses can be described using the presented model that is based on the underlying physical effect (Mie-scattering) identified as the most important factor (in soiling loss for low soiling levels). This model holds true for the experimental data (natural outdoor soiling) for both technologies CSP and PV and thus supports the presented approach and the results. Furthermore, the influence of angles of incidence (AOI) on Cleanliness measurements is investigated. It is shown, that measurements taken under normal AOI do not necessarily reflect the effects of soiling on the induced energy loss well in an annual frame of reference. Suggestions are made to measure the Cleanliness under various AOIs with a corresponding weighting pattern.

\section{Acknowledgements}

R. Conceição is grateful to the Renewables Energies Chair and Institute of Earth Sciences, University of Évora, as well as, to the Infraestrutura Nacional de Investigação em Energia Solar de Concentração (INIESC).

\section{References}


[1] B. Figgis, A. Ennaoui, S. Ahzi, Y. Remond, Review of PV soiling particle mechanics in desert environments, Renewable and Sustainable Energy Reviews 76 (2017) 872-881. doi:10.1016/j.rser.2017.03.100.

[2] S. Ghazi, A. Sayigh, K. Ip, Dust effect on flat surfaces: A review paper, Renewable and Sustainable Energy Reviews 33 (2014) 742-751. doi:10.1016/j.rser.2014.02.016.

[3] L. L. Kazmerski, M. A. Jardan, Y. A. Jnoobi, Y. A. Shaya, J. J. John, Ashes to ashes, dust to dust: Averting a potential showstopper for solar photovoltaics, in: 2014 IEEE 40th Photovoltaic Specialist Conference (PVSC), IEEE, 2014. doi:10.1109/pvsc.2014.6925524.

[4] T. Sarver, A. Al-Qaraghuli, L. L. Kazmerski, A comprehensive review of the impact of dust on the use of solar energy: History, investigations, results, literature, and mitigation approaches, Renewable and Sustainable Energy Reviews 22 (2013) 698-733. doi:10.1016/j.rser.2012.12.065.

[5] S. C. Costa, A. S. A. Diniz, L. L. Kazmerski, Dust and soiling issues and impacts relating to solar energy systems: Literature review update for 2012-2015, Renewable and Sustainable Energy Reviews 63 (2016) 33-61. doi:10.1016/j.rser.2016.04.059.

[6] J. J. John, Characterization of soiling loss on photovoltaic modules, and development of a novel cleaning system, Phd thesis, Indian Institute of Technology Bombay (2015). URL: https://www.ee.iitb.ac.in/ anilkg/Jim-thesis.pdf (last visit on 2019-11-18).

[7] R. Conceição, H.G. Silva, J. Mirão, M. Collares-Pereira, Organic soiling: The role of pollen in PV module performance degradation, Energies 11 (2) (2018) 294. doi:10.3390/en11020294.

[8] E. Roth, R. Pettit, The effect of soiling on solar mirrors and techniques used to maintain high reflectivity (this work is supported by the division of solar technology, U.S. department of energy (DOE), under contract DE-AC04-76- DP00789) (1980) 199-227. doi:10.1016/b978-0-12511160-7.50013-2.

[9] R. Pettit, J. Freese, Wavelength dependent scattering caused by dust accumulation on solar mirrors, Solar Energy Materials 3 (1-2) (1980) 1-20.doi:10.1016/0165-1633(80)90046-5.

[10] A. Heimsath, P. Lindner, E. Klimm, T. Schmid, K. O. Moreno, Y. Elon, M. Am-Shallem, P. Nitz, Specular reflectance of soiled glass mirrors - study on the impact of incidence angles, Author(s), 2016. doi:10.1063/1.4949219. 
[11] A. Heimsath, T. Schmid, P. Nitz, Angle resolved specular reflectance measured with VLABS, Energy Procedia 69 (2015) 1895-1903. doi:10.1016/j.egypro.2015.03.173.

[12] R. Conceição, H. G. Silva, L. Fialho, F. M. Lopes, M. Collares-Pereira, PV system design with the effect of soiling on the optimum tilt angle, Renewable Energy 133 (2019) 787-796. doi:10.1016/j.renene.2018.10.080.

[13] F. Wolfertstetter, K. Pottler, A novel method for automatic real-time monitoring of mirror soiling rates, DLR, 2012.

[14] E. P. Roth, A. J. Anaya, The effect of natural soiling and cleaning on the size distribution of particles deposited on glass mirrors, Journal of Solar Energy Engineering 102 (4) (1980) 248. doi:10.1115/1.3266188.

[15] B. Figgis, Pv soiling: Qatar perspective, in: DEWA Soilingworkshop 2016, 2016.

[16] R. Wagner, T. Ajtai, K. Kandler, K. Lieke, C. Linke, T. M"uller, M. Schnaiter, M. Vragel, Complex refractive indices of saharan dust samples at visible and near UV wavelengths: a laboratory study, Atmospheric Chemistry and Physics 12 (5) (2012) 2491-2512. doi:10.5194/acp-12-2491-2012.

[17] P. Laven, MiePlot (2017). URL: http://www.philiplaven.com/mieplot.htm (last visit 201911-18)

[18] F. Wolfertstetter, Auswirkungen von verschmutzung auf konzentrierende solarthermische kraftwerke, PhD thesis, Technische Hochschule Aachen (2016). URL: http://publications.rwthaachen.de/record/706465/files/706465.pdf (last visit 2019-11-18)

[19] B. Knisely, S. V. Janakeeraman, J. Kuitche, G. TamizhMani, Validation of draft international electrotechnical commission 61853-2 standard: Angle of incidence effect on photovoltaic modules, Tech. rep., Arizona State University Photovoltaic Reliability Laboratory (2013).

[20] N. Mart'in, J. M. Ruiz, A new model for PV modules angular losses under field conditions, International Journal of Solar Energy 22 (1) (2002) 19-31. doi:10.1080/01425910212852. 
[21] N. MartAn, F. Chenlo, E. Mejuto, F. Soriano, Validating an angular of incidence losses model with different pv technologies and soiling conditions, in: European Photovoltaic Solar Energy Conference and Exhibition, Vol. 27th, 2012. 17

[22] N. Janotte, Requirements for representative acceptance tests for the prediction of the annual yield of parabolic trough solar fields, PhD thesis, Technische Hochschule Aachen (2012). URL: https://elib.dlr.de/79184/ (last visit 2019-11-18)

[23] C. Baltus, J. Eikelboom, R. van Zolingen, Analytical monitoring of losses in PV systems, Vol. 14, Netherlands Energy Research Foundation ECN, 1997. 18

[24] Zorrilla-Casanova, J., Piliougine, M., Carretero, J., Bernaola-Galván, P., Carpena, P., MoraLópez, L., Sidrach-de-Cardona, M., 2012. Losses produced by soiling in the incoming radiation to photovoltaic modules. Progress in Photovoltaics: Research and Applications n/a-n/a. https://doi.org/10.1002/pip.1258

[24] J. R. Caron and B. Littmann, Direct Monitoring of Energy Lost Due to Soiling on First Solar Modules in California, IEEE Journal of Photovoltaics, vol. 3, no. 1, pp. 336-340, 2013. [25] C. P. Ryan, F. Vignola, D. K. McDaniels, "Solar cell arrays: degradation due to dirt", Proc. of the American Section of the International Solar Energy Society, pp. 234-237, Denver,

[26] R. Conceição, A. Merrouni, D. Lopes, A. Alae, H.G. Silva, El-G. Bennouna, M. Collares Pereira, A. Ghennioui, A comparative study of soiling on solar mirrors in Portugal and Morocco: Preliminary results for the dry season, SolarPACES 2018, 2126, 220001-1-220001-7

[27] R. Conceição, Soiling in Solar Energy Conversion Technologies: Assessment and Mitigation, PhD Thesis, 2019, University of Évora. URL:

http://dspace.uevora.pt/rdpc/handle/10174/25527 (last visit 2019-11-18) 